\title{
Asymptomatic intracranial hemorrhage in a newborn with congenital factor VII deficiency and successful treatment with recombinant activated factor VII
}

\author{
Zeynep İnce ${ }^{1}$, Özgül Bulut ${ }^{1}$, Melike Tuğrul-Aksakal ${ }^{1}$, Ayşegül Ünüvar ${ }^{2}$, Ömer Devecioğlu², \\ Asuman Çoban ${ }^{1}$ \\ Divisions of ${ }^{1}$ Neonatology and ${ }^{2}$ Hematology and Oncology, Department of Pediatrics, Istanbul University İstanbul Faculty \\ of Medicine, İstanbul, Turkey. E-mail: ozgulbulut@yahoo.com \\ Received: 26th September 2017, Accepted: 30th October 2017
}

\begin{abstract}
SUMMARY: İnce Z, Bulut Ö, Tuğrul-Aksakal M, Ünüvar A, Devecioğlu Ö, Çoban A. Asymptomatic intracranial hemorrhage in a newborn with congenital factor VII deficiency and successful treatment with recombinant activated factor VII. Turk J Pediatr 2018; 60: 562-565.

Intracranial hemorrhage is considered the most common cause of death in newborns with congenital factor VII (FVII) deficiency. Recombinant activated FVII (rFVIIa) provides specific replacement therapy, however there is limited experience with its neonatal use. We describe our experience about the treatment of intracranial hemorrhage in a newborn with congenital FVII deficiency and emphasize the importance of imaging in asymptomatic patients. She presented with ecchymoses on her skin, no other pathological clinical signs, prolonged PT, normal PTT and FVII activity of $2 \%$. Intracranial hemorrhage was diagnosed while screening for internal bleedings. Treatment with rFVIIa resulted in stabilization and regression of the hematoma.
\end{abstract}

Key words: factor VII deficiency, intracranial hemorrhage, newborn, recombinant activated factor VII.

Congenital factor VII (FVII) deficiency is a rare autosomal-recessive bleeding disorder which is characterized by a variable clinical presentation ranging from asymptomatic disease to severe bleeding episodes ${ }^{1}$. Intracranial hemorrhage occurs in approximately $4 \%$ of affected patients and is considered the most common cause of death in newborns with FVII deficiency. ${ }^{2}$ Replacement therapy with fresh frozen plasma (FFP) in newborns carries inherent dangers of infectious complications and fluid overload. Recombinant activated FVII (rFVIIa) provides specific factor replacement with much less risk, however there is limited experience with its use in the neonatal period.

In this report, we describe the successful management of intracranial hemorrhage in a newborn with congenital factor VII deficiency and treatment with rFVIIa.

\section{Case Report}

The patient was a full-term female product of an uncomplicated pregnancy with normal vaginal delivery. It was the first pregnancy of the mother. The parents were first degree cousins. There was no family history of a bleeding disorder. After an uneventful postnatal adaptation, jaundice was observed when the baby was three days old. The bilirubin levels peaked on the sixth postnatal day and then decreased to normal levels without treatment. On the seventh day of life, bruises on her skin (leg and abdomen) were noted by the parents, however, they did not seek any medical advice. When the lesions persisted, they brought the baby to our hospital on the tenth postnatal day and she was admitted. On physical examination, she had ecchymotic areas on her abdomen and left leg which were fading. Her activity was good and she had no other abnormal physical findings.

The initial laboratory studies were as follows: hemoglobin $(\mathrm{Hb}) 10.2 \mathrm{~g} / \mathrm{dl}$, hematocrit (Hct) $27.2 \%$, white blood cell count 11,500/ $\mu 1$, platelet count $649,000 / \mathrm{mm}^{3}$, normal erythrocyte morphology, prothrombin time 
(PT) 54 sec., activated partial thromboplastin time (aPTT) 31.7 sec., INR: 4.71. To rule out vitamin $\mathrm{K}$ deficiency, $2 \mathrm{mg}$ vitamin $\mathrm{K}$ was given intravenously. After four hours, control values were as follows: PT $57.8 \mathrm{sec}$., aPTT $33.1 \mathrm{sec}$, INR 5.06. Although there were no clinical signs and symptoms, cranial and abdominal imaging was ordered to rule out internal organ bleeding. Cranial ultrasonography (US) showed intracranial hemorrhage (ICH) which was confirmed by cranial computerized tomography (CT) with parenchymal bleeding in the left parieto-occipital area (Fig. 1). After taking plasma samples for coagulation factor activity, fresh frozen plasma (FFP) was given $(10 \mathrm{ml} /$ $\mathrm{kg})$. After the transfusion, control values were PT $25.5 \mathrm{sec}$. and aPTT $37 \mathrm{sec}$. The laboratory results for factor activities showed severe $\mathrm{F}$ VII deficiency with an activity of $2 \%$. The other factor activities were as follows: FII $88 \%$, FVIII $175 \%$, FIX 90\%, FX 69\%, FXI $74 \%$. After consultation with the Division of Pediatric Hematology and Oncology, treatment with activated recombinant FVII (rFVIIa) was started. An initial dose of rFVIIa $30 \mu \mathrm{g} / \mathrm{kg}$ was given and then continued with a dosing schedule of $15 \mu \mathrm{g} / \mathrm{kg}$ every 6 hours. FVII levels before and after rFVIIa infusion were monitored and as this lower dose did not produce acceptable levels of FVII, the dose was increased to $30 \mu \mathrm{g} / \mathrm{kg}$ every 6 hours and continued for 3 days. Then the dose was decreased to $20 \mu \mathrm{g} / \mathrm{kg}$ every 6 hours which maintained sufficient levels of FVII and continued for three weeks. The patient was consulted with the Department of Neurosurgery and no surgical intervention was undertaken. Stabilization and regression of the hematoma was observed on serial imaging by US and CT (Fig. 2). The baby was discharged on 24th day of the treatment. Prophylaxis with rFVIIa and follow-up by the Division of Pediatric Hematology and Oncology was planned. No adverse events have been observed in the patient during the treatment period.

Written consent was obtained from parents of patient before submission.

\section{Discussion}

Factor VII deficiency is a rare bleeding disorder that is transmitted as an autosomal recessive trait. Its prevalence is equal among males and females and is estimated at 1:500,000, accounting for approximately $0.5 \%$ of all inherited coagulation disorders. ${ }^{3}$ A prolonged PT with normal aPTT indicates FVII deficiency. For definitive diagnosis, specific plasma FVII levels should be measured.

FVII deficiency is expressed in different ways and leads to various clinical pictures. In congenital FVII deficiency, bleeding can occur during any period of life and can either be diagnosed at childhood or adulthood. There is a poor correlation between FVII coagulant activity and clinical bleeding tendency. ${ }^{4}$ The expression of this disease is highly variable. Factor levels between 10 and $25 \%$ have been described as sufficient for adequate hemostasis ${ }^{5}$. In our case, the patient presented with ecchymoses on her skin and intracranial hemorrhage was diagnosed while investigating for internal organ bleeding. Her FVII level was $2 \%$ and prolonged PT did not improve with vitamin $\mathrm{K}$ administration.

A dose of $1 \mathrm{IU} / \mathrm{kg}$ factor VII is known to produce a $1.9 \%$ rise in plasma FVII activity. ${ }^{6}$ Recombinant FVIIa has been demonstrated in congenital FVII-deficient patients, primarily in short-term surgical prophylaxis and in the management of acute bleeding episodes such as gum bleeding, hemarthrosis and intracranial hemorrhage. $5,7,8$

There is only limited data about the neonatal use of rFVIIa and most of the reports are about the use of this product in critical lifethreatening hemorrhages in newborn and preterm infants. The doses used for this purpose are higher than those used for congenital FVII deficiency. Brady et al. ${ }^{9}$, reported that activated factor VIIa is a powerful hemostatic agent that apparently successfully stopped acute critical hemorrhage in nine newborn patients. Olomu et al. ${ }^{10}$ reported on two very low birth weight infants who received rFVIIa at a dose of $50 \mu \mathrm{g} / \mathrm{kg}$ every $3 \mathrm{~h}$ for $2-3$ days for severe pulmonary hemorrhage, after failure of standard therapies. Griesen et al. ${ }^{11}$ suggested that the half-life of rFVIIa in preterm babies is within the range of 2-3 h. In their series, rFVIIa was also well tolerated. Veldman et al. ${ }^{12}$ reported on 10 preterm infants between 23 and 28 weeks of gestation who were prophylactically administered $100 \mu \mathrm{g} / \mathrm{kg}$ rFVIIa bolus injection within the first $2 \mathrm{~h}$ of life, followed by $100 \mu \mathrm{g} /$ $\mathrm{kg}$ every $4 \mathrm{~h}$, for the first $72 \mathrm{~h}$ of life. None had any complication like venous thrombosis, 


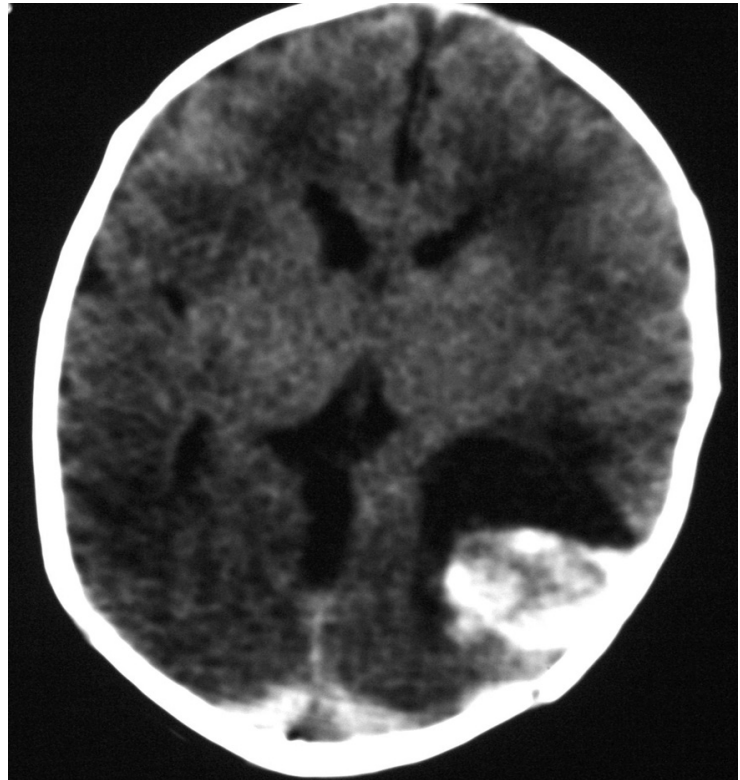

Fig. 1. Cranial computerized tomography at admission showing parenchymal bleeding in the left parieto-occipital area.

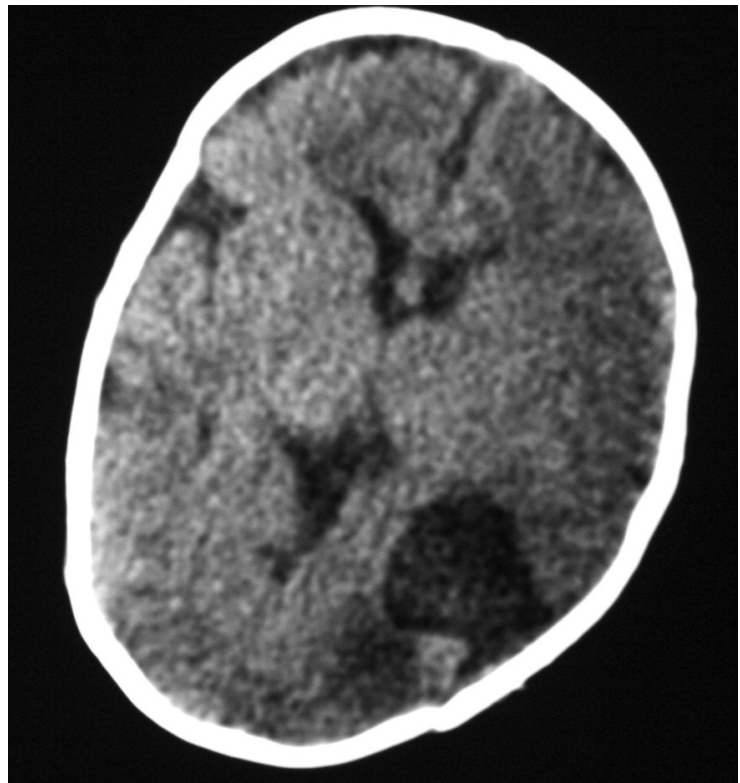

Fig. 2. Cranial computerized tomography showing stabilization and regression of the intracranial hematoma after treatment with rFVIIa.

cerebral infarction, platelet consumption and DIC. However, the authors concluded that in that pilot study, which did not provide the sample size to assess any effect of rFVIIa on the incidence of intraventricular hemorrhage (IVH), $20 \%$ of the neonates went on to have grade III or IV IVH, which was similar to the rate in studies in which rFVIIa was not given.
Although there is only mid-level evidence for the use of rFVII in congenital FVII deficiency, it has been used as an effective replacement product to treat these patients and case reports support the efficacy and safety of rFVIIa. ${ }^{8,13,14}$ The experience on the use of rFVIIa in newborn cases with congenital FVII deficiency is very limited. The study by Brenner and Wiis ${ }^{14}$, reviewed 39 cases in which rFVIIa has been used to treat or prevent bleeding episodes in a total of 30 patients with congenital FVII deficiency. The data showed that, in most cases, a low median dose of rFVIIa $(13.3 \mu \mathrm{g} / \mathrm{kg})$ was effective in achieving hemostasis. They suggested that the recommended dosage of $15-30 \mu \mathrm{g} / \mathrm{kg}$ every 4-6 h for FVII deficiency (compared with $90 \mu \mathrm{g} / \mathrm{kg}$ for both hemophilia with inhibitors and Glanzmann's thrombasthaenia) was appropriate when treating bleeds associated with a deficiency in FVII. In this report, there was only one case of disseminated intravascular coagulation (DIC) in a premature infant. There were no other newborn cases.

In the case report by Farah et al. ${ }^{1}$, relatively low doses of rFVIIa were effective in preventing future life-threatening bleeding episodes in a newborn patient with severe FVII deficiency. They administered $60 \mu \mathrm{g} / \mathrm{kg}$ once and then $30 \mu \mathrm{g} / \mathrm{kg}$ every $4-6 \mathrm{~h}$ for 10 days, then once a day for 5 days.

The review by Mariani et al. ${ }^{8}$ reported the use of rFVIIa in children as prophylaxis for $\mathrm{ICH}$, recurrent epistaxis, menorrhagia and hemarthrosis. The dosing regimens were in the range of $15-90 \mu \mathrm{g} / \mathrm{kg}$ by bolus injection from twice daily to twice weekly for up to 2 years. All regimens resulted in a reduced number of spontaneous bleeds or an absence of bleeding problems, although no information was reported on the severity of observed bleeds for which prophylaxis was instituted. There were no newborn cases in this review.

Another case report by Wong et al. ${ }^{7}$ emphasized that the use of replacement FVII is critical to the successful treatment of life-threatening bleeds in newborns and infants with severe FVII deficiency. In their case, rFVIIa was welltolerated, maintained effective hemostasis with good clinical outcome, and produced consistent therapeutic mean trough levels above $25 \%$ FVII concentration, even at $15 \mu \mathrm{g} / \mathrm{kg}$ every 4 hours.

Karimi and Shafieian ${ }^{15}$ reported two cases of 
newborn infants with severe FVII deficiencies and the use of prophylactic treatment with rFVIIa with a dosage of $20-30 \mu \mathrm{g} / \mathrm{kg}$ once to twice per week. They suggested that this may be beneficial in preventing hemorrhagic complications, particularly $\mathrm{ICH}$ and brain damage, especially in infants, in spite of short plasma half-life (3-6 h).

In our case a dosing schedule of $30 \mu \mathrm{g} /$ kg rFVIIa every 6 hours for 3 days initially, followed by $20 \mu \mathrm{g} / \mathrm{kg}$ every 6 hours for three weeks maintained sufficient levels of FVII with resultant stabilization and regression of the intracranial hematoma.

Our case indicates that some neonatal patients with severe FVII deficiency may present with minimal symptoms or be asymptomatic. In case of a prolonged PT but normal PTT, plasma FVII activity should be measured and cranial imaging should be done because of intracranial hemorrhage risk. Considering the use of rFVIIa in FVII deficient newborns, adopted treatment regimens typically involve frequent dosing intervals every 3-6 h, as rFVIIa has a short half-life similar to FVII. Further studies are necessary to assess the optimal dosing regimens, consequences of long-term rFVIIa therapy and the long-term clinical outcomes of severely FVII-deficient patients on prophylaxis.

\section{REFERENCES}

1. Farah RA, Hamod D, Melick N, Giansily-Blaizot M, Sallah S. Successful prophylaxis against intracranial hemorrhage using weekly administration of activated recombinant factor VII in a newborn with severe factor VII deficiency. J Thromb Haemost 2007; 5: 433-434.

2. Mariani G, Herrmann FH, Dolce A, et al; International Factor VII Deficiency Study Group. Clinical phenotypes and factor VII genotype in congenital factor VII deficiency. Thromb Haemost 2005; 93: 481-487.

3. Williams WJ, Beutler E, Erslev AJ and Lichtman MA. Hematology (3rd ed), New York: McGraw-Hill, 1983.

4. Cooper DN, Millar DS, Wacey A, Banner DW, Tuddenham EG. Inherited factor VII deficiency: Molecular genetics and pathophysiology. Thromb Haemost 1997; 78: 151-160.
5. Ingerslev J, Kristensen HL. Clinical picture and treatment strategies in factor VII deficiency. Haemophilia 1998; 4: 689-696

6. Charpiat B, Laporte S, Mismetti P, Debize G, Ducerf C. Perioperative pharmacokinetics of factor VII concentrate during liver surgery in a patient with congenital factor VII deficiency: An individual mathematical model. Blood Coagul Fibrinolysis 2002; 13: 457-460.

7. Wong WY, Huang WC, Miller R, McGinty K, Whisnant JK. Clinical efficacy and recovery levels of recombinant FVIIa (NovoSeven) in the treatment of intracranial haemorrhage in severe neonatal FVII deficiency. Haemophilia 2000; 6: 50-54.

8. Mariani G, Testa MG, Di Paolantonio T, Molskov Bech $\mathrm{R}$, Hedner U. Use of recombinant, activated factor VII in the treatment of congenital factor VII deficiencies. Vox Sang 1999; 77: 131-136.

9. Mathew P, Young G. Recombinant factor VIIa in paediatric bleeding disorders -a 2006 review. Haemophilia 2006; 12: 457-472.

10. Brenner B, Wiis J. Experience with recombinantactivated factor VII in 30 patients with congenital factor VII deficiency. Hematology 2007; 12: 55-62.

11. Greisen G, Andreasen RB. Recombinant factor VIIa in preterm neonates with prolonged prothrombin time. Blood Coagul Fibrinolysis 2003; 14: 117-120.

12. Brady KM, Easley RB, Tobias JD. Recombinant activated factor VII (rFVIIa) treatment in infants with hemorrhage. Pediatr Anaesth 2006; 16: 1042-1046.

13. Olomu N, Kulkarni R, Manco-Johnson M. Treatment of severe pulmonary hemorrhage with activated recombinant factor VII (rFVIIa) in very low birth weight infants. J Perinatol 2002; 22: 672-674.

14. Karimi M, Shafieian R. Prophylactic effect of recombinant factor VIIa with congenital factor VII deficiency. Haemophilia 2008; 14: 851-852.

15. Veldman A, Josef J, Fischer D, Volk WR. A prospective pilot study of prophylactic treatment of preterm neonates with recombinant activated factor VII during the first 72 hours of life. Pediatr Crit Care Med 2006; 7: 34-39. 